\title{
Magnetic survey and ChinaGRF 2000
}

\author{
Wen-Yao Xu ${ }^{1}$, Guo-Hui Xia ${ }^{1}$, Zhen-Chang $\mathrm{An}^{1}$, Geng-Xiong Chen ${ }^{1}$, Feng-Yu Zhang ${ }^{1}$, Yue-Hua Wang ${ }^{1}$, \\ Yu-Gang Tian ${ }^{1}$, Zi-Gang $\mathrm{Wei}^{1}$, Shi-Zhuang $\mathrm{Ma}^{2}$, and Hong-Fei Chen ${ }^{3}$ \\ ${ }^{1}$ Institute of Geology and Geophysics, Chinese Academy of Sciences, Beijing, China \\ ${ }^{2}$ Graduate School, Chinese Academy of Sciences, Beijing, China \\ ${ }^{3}$ Peking University, Beijing, China
}

(Received December 18, 2002; Revised March 4, 2003; Accepted March 24, 2003)

\begin{abstract}
The magnetic survey in China carried out in resent years is briefly introduced. On the basis of the magnetic data at 119 repeat stations, 39 permanent observatories and 20 grid values of the IGRF 2000, the magnetic charts and models of China for 2000, called China Geomagnetic Reference Field 2000 (or ChinaGRF 2000) have been compiled.
\end{abstract}

Key words: Magnetic survey, Magnetic chart, ChinaGRF.

\section{Introduction}

From the beginning of twentieth century, magnetic survey has been carried out in China. The survey was confined in southeast and southwest china, mainly along Yangtze River, in Hainan Island and Xisha Islands. The measured magnetic data during 30 years from 1906 to 1936 were used to compile regional magnetic charts for 1909-1915, 19151920, 1920-1930, and 1930-1936 (Chen and Liu, 1948; Liu and Li, 1948; Chen, 1949). From fifties of 20th century the countrywide magnetic survey has been carried out, and the network of magnetic repeat stations has been gradually built up. The stations are reoccupied every 10 years on average, and repeat station observations were made for $D, H$ and $F$, or $D, I$ and $F$. Magnetic charts for 1950, 1960, 1970, 1980, and 1990 were compiled. At the same time, magnetic models have been established (Xia et al., 1988; Wang et al., 1999; An, 2001).

In order to compile magnetic charts for 2000 and study the secular variations in the magnetic field, a magnetic survey was made in China for 1998-2001. A new generation of China magnetic charts was compiled by using these data and the data from permanent magnetic observatories and the IGRF 2000.

\section{Magnetic Survey in China during 1998-2001}

A field survey of the magnetic field was carried out during 1998-2001. Declination $(D)$, inclination $(I)$ and total intensity $(F)$ were measured by DIM-100 and CZM-2 proton precession magnetometers at 119 repeat stations, which are fairly well distributed all over the country, as shown by open circles in the top left panel of Fig. 1. The accuracies of the instruments are $\pm 0^{\prime} .1$ for DIM-100 and $1.5 \mathrm{nT}$ for proton precession magnetometer. Typical uncertainties in field values at the repeat stations are $\pm 0^{\prime} .3, \pm 0^{\prime} .3$ and $\pm 1.5 \mathrm{nT}$ for $D, I$ and $F$, respectively.

Copy right(c) The Society of Geomagnetism and Earth, Planetary and Space Science (SGEPSS); The Seismological Society of Japan; The Volcanological Society of Japan; The Geodetic Society of Japan; The Japanese Society for Planetary Sciences.
Repeat station data are processed following normal practice. Thirty-nine permanent magnetic observatories shown in the top left panel of Fig. 1 by solid squares are used for the data reduction. The diurnal variations and other disturbances at the repeat stations are eliminated by reference to the nearest magnetic observatories, and the station values are updated to a common epoch (2000.0) by using the hourly values at the reference observatories. Other 4 elements of the geomagnetic field $(H, X, Y$, and $Z)$ are derived from $D$, $I$, and $F$.

The secular variation at a repeat station is estimated from the gradient of a plot of the repeat station data vs. time.

\section{China Geomagnetic Reference Field 2000}

The reduced data are used to establish magnetic models and compile magnetic charts for the normal geomagnetic field and its secular variation. Several methods of modeling the magnetic field have been tested. The results from different methods are generally in good agreement. In this paper the Taylor model is introduced, which is produced by using Taylor polynomial filled by the method of least squares at different truncation level,

$$
B(\phi, \lambda)=\sum_{i=0}^{N} \sum_{j=0}^{N-i} a_{i j}\left(\phi-\phi_{0}\right)^{i}\left(\lambda-\lambda_{0}\right)^{j}
$$

where $B(\phi, \lambda)$ is magnetic element, $\phi$ and $\lambda$ are latitude and longitude of stations, $\phi_{0}$ and $\lambda_{0}$ are the coordinates of the origin for Taylor series expansion, $N$ is truncation level. In the present case, $\phi_{0}=36^{\circ}$ and $\lambda_{0}=106^{\circ}$ are adopted. The number of the Taylor coefficients $a_{i j}$ is $(N+1)(N+2) / 2$. In the computation the unit of radian is used to latitude, longitude, declination, and inclination.

After analyzing and comparing the errors for truncation levels $N=1-10$, the most appropriate truncation level $N=4$ was selected to represent the normal magnetic field in China for 2000 and the secular variation for 2000-2005 (An, 2001). Each of elements of the field is modeled independently. 


\section{MAGNETIC CHARTS OF CHINA FOR 2000}

\section{o repeat station - permanent station + IGRF}
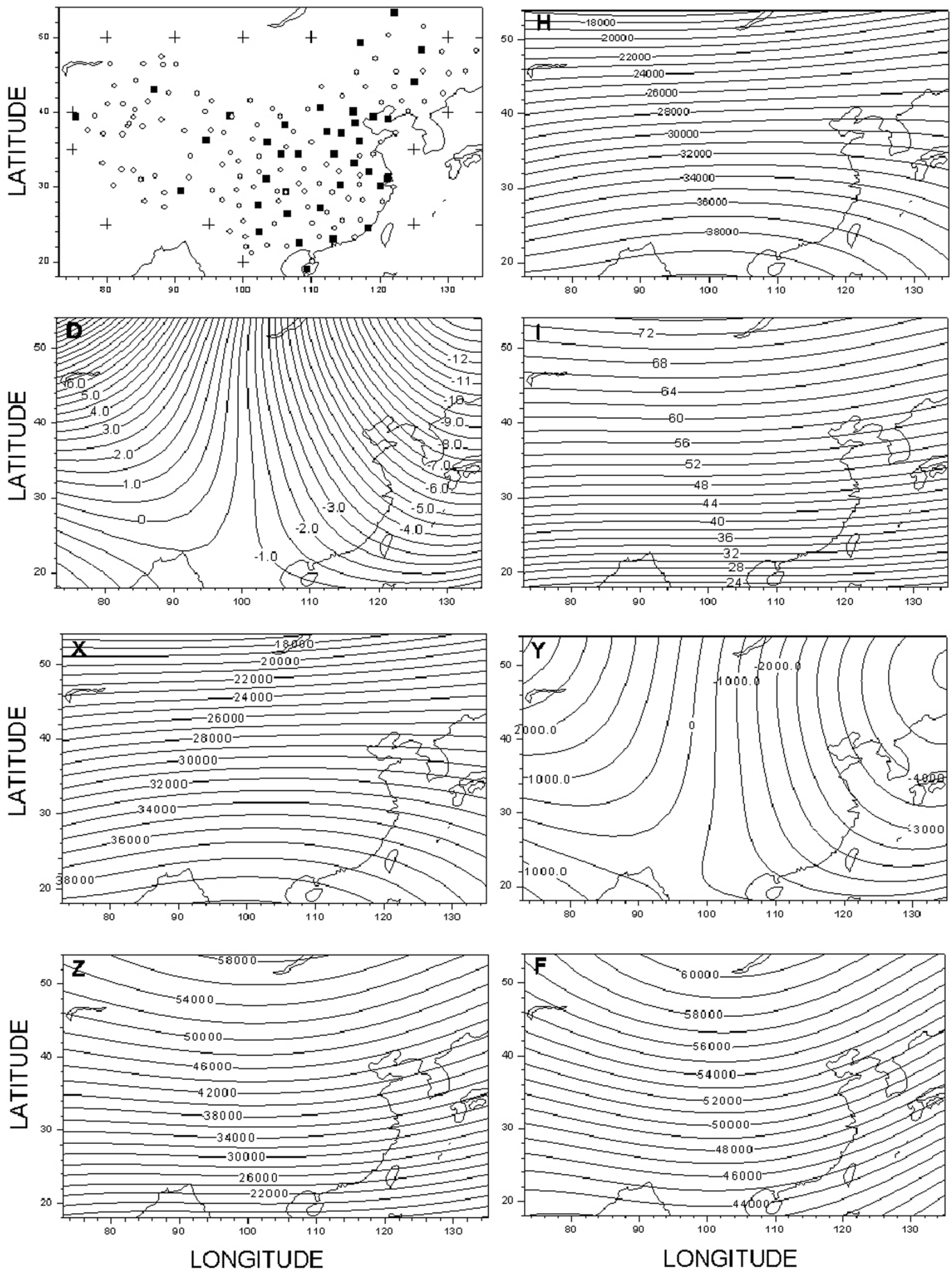

Fig. 1. Magnetic charts of China for 2000. 
Table 1. Taylor coefficients of the ChinaGRF 2000.

\begin{tabular}{|c|c|c|c|c|c|c|c|}
\hline \multirow{2}{*}{\multicolumn{2}{|c|}{$a_{i j}$}} & \multicolumn{6}{|c|}{$i$} \\
\hline & & & & 1 & 2 & 3 & 4 \\
\hline \multirow{15}{*}{$j$} & \multirow[t]{3}{*}{0} & $D(\mathrm{rad})$ & -0.02924 & -0.05452 & -0.08668 & -0.29068 & 1.17998 \\
\hline & & $I(\mathrm{rad})$ & 0.85938 & 1.49132 & -1.35051 & 0.50450 & 1.86202 \\
\hline & & $F(\mathrm{nT})$ & 51193.2 & 33608.8 & -5721.7 & -53863.7 & 36385.6 \\
\hline & \multirow[t]{3}{*}{1} & & -0.21126 & -0.74030 & -0.17534 & -0.73940 & \\
\hline & & & -0.02815 & -0.12667 & 0.08175 & -0.09373 & \\
\hline & & & -1718.2 & -633.2 & 1166.0 & 21852.0 & \\
\hline & \multirow[t]{3}{*}{2} & & -0.13160 & 0.15822 & 0.96224 & & \\
\hline & & & -0.13519 & -0.23585 & -0.01428 & & \\
\hline & & & -16218.9 & -10962.4 & 28949.3 & & \\
\hline & \multirow[t]{3}{*}{3} & & 0.29633 & 0.39365 & & & \\
\hline & & & -0.12203 & 0.37305 & & & \\
\hline & & & -4481.8 & 11400.2 & & & \\
\hline & 4 & & 0.23213 & & & & \\
\hline & & & 0.14776 & & & & \\
\hline & & & 10158.4 & & & & \\
\hline
\end{tabular}

Table 2. Taylor coefficients of the secular variation model for 2000-2005.

\begin{tabular}{|c|c|c|c|c|c|c|c|}
\hline \multirow{2}{*}{\multicolumn{2}{|c|}{$a_{i j}$}} & \multicolumn{6}{|c|}{$i$} \\
\hline & & & & 1 & 2 & 3 & 4 \\
\hline \multirow{15}{*}{$j$} & \multirow[t]{3}{*}{0} & $D(\mathrm{rad} / \mathrm{a})$ & 0.000037 & 0.000090 & -0.001686 & -0.005104 & -0.005042 \\
\hline & & $I(\mathrm{rad} / \mathrm{a})$ & 0.000905 & -0.001439 & 0.001891 & 0.000889 & -0.008332 \\
\hline & & $F(\mathrm{nT} / \mathrm{a})$ & 23.94 & -20.86 & -15.89 & 37.70 & -217.80 \\
\hline & \multirow[t]{3}{*}{1} & & -0.001709 & -0.000385 & 0.004997 & -0.000264 & \\
\hline & & & -0.000151 & 0.004737 & -0.005695 & -0.016141 & \\
\hline & & & 4.75 & -62.13 & -196.14 & 266.41 & \\
\hline & \multirow[t]{3}{*}{2} & & 0.000977 & -0.000529 & 0.015446 & & \\
\hline & & & -0.002320 & -0.000471 & 0.005330 & & \\
\hline & & & -9.16 & 107.44 & -95.23 & & \\
\hline & \multirow[t]{3}{*}{3} & & 0.003289 & -0.005674 & & & \\
\hline & & & 0.001397 & -0.007588 & & & \\
\hline & & & -9.26 & 108.21 & & & \\
\hline & \multirow[t]{3}{*}{4} & & -0.003834 & & & & \\
\hline & & & 0.004740 & & & & \\
\hline & & & 19.81 & & & & \\
\hline
\end{tabular}

In order to improve the boundary condition, the International Geomagnetic Reference Field 2000 (IGRF 2000) with the maximum degree 10 was used to create the magnetic field values at 20 positions near the boundary regions, as shown by cross in the top left panel of Fig. 1 .

The root-mean-square deviations between the model and station values are $11^{\prime} .88$ for declination, $13^{\prime} .35$ for inclination, and $131.6 \mathrm{nT}$ for total intensity, respectively. The Taylor coefficients for the truncation level $N=4$ are listed in Table 1. The magnetic charts are shown in Fig. 1, in which the contour intervals are $0.5^{\circ}, 2^{\circ}, 1000 \mathrm{nT}, 500 \mathrm{nT}, 2000 \mathrm{nT}$, $1000 \mathrm{nT}$, and $1000 \mathrm{nT}$ for $D, I, X, Y, Z, H$, and $F$, respectively.

The secular variation models for 2000-2005 were also established, which are represented by a Taylor polynomial with truncation level 4 , the coefficients of which are listed in Table 2.

The magnetic anomalies in China were examined by comparing the IGRF model values and the measured components at 37 permanent observatories (Wang, 2003). The root-mean square deviation between the observed field and IGRF model is $202.3 \mathrm{nT}$ for total intensity, which is much greater than that for ChinaGRF 2000. Accordingly, the regional mag- netic field model ChinaGRF obtained in this paper can be used to describe the field distribution in China region with better approximation than IGRF.

\section{References}

An, Z.-C., Analysis and discussion of the geomagnetic field polynomial models, Chinese J. Geophys., 44(supplement), 45-50, 2001.

Chen, Z.-Q., A detailed geomagnetic survey of Pehpei district, Szechuan, China, Acta Geophys., 1, 177-186, 1949.

Chen, Z.-Q. and Q.-L. Liu, Preliminary report on the results of geomagnetic survey in China, 1946-1947, Acta Geophys., 1, 78-87, 1948.

Liu, Q.-L. and S.-B. Li, Geomagnetic survey in southwestern China, 19401943, Acta Geophys., 1, 68-77, 1948.

Wang, T.-W., Analysis on the errors of IGRF models in China area, Chinese J. Geophys., 46(2), 2003 (in press).

Wang, Y.-H, Z.-C. An, V. P. Golovkov, N. M. Rotanova, and A. L. Kharitonov, Theoretical analysis of geomagnetic field over east Asia and rectangular harmonic model,Chinese J. Geophys., 42(5), 640-647, 1999.

Xia, G.-H., S.-L. Zheng, L.-L. Wu, F.-Y. Zhang, and H. Wei, The geomagnetic field chart of China in 1980.0 and the mathematic model, Acta Geophys., 31, 82-89, 1988.

W.-Y. Xu (e-mail: wyxu@ mail.igcas.ac.cn), G.-H. Xia, Z.-C. An, G.-X Chen, F.-Y. Zhang, Y.-H. Wang, Y.-G. Tian, Z.-G. Wei, S.-Z. Ma, and H.-F. Chen 